\title{
CDISC ADaM Analysis Reason Terminology
}

National Cancer Institute

\section{Source}

National Cancer Institute. CDISC ADaM Analysis Reason Terminology. NCI Thesaurus. Code $C 117744$.

Terminology associated with the analysis reason codelist of the Clinical Data Interchange Standards Consortium (CDISC) Analysis Data Model (ADaM). 